\title{
Unresolved nature source TeV J2032+4130
}

\author{
V. G. Sinitsyna ${ }^{1, *}, K$. A. Balygin ${ }^{1,2}, S . S$. Borisov $^{1}, A . I . \mathrm{Klimov}^{1,2}, R . M$. Mirzafatikhov $^{1}$, \\ N. I. Moseiko $^{2}, I$. E. Ostashev ${ }^{1,2}$, and V. Y. Sinitsyna ${ }^{1}$ \\ ${ }^{1}$ P.N. Lebedev Physical Institute, Moscow, Russia \\ ${ }^{2}$ NRC "Kurchatov Institute”, Moscow, Russia
}

\begin{abstract}
Cygnus Region contains many objects that are bright in all wavelengths, including one of the most powerful active star formation regions: Cyg OB2, pulsars, and supernova remnants. Some of the sources have been detected at high and very high energies. One of them was discovered due to the proximity to well-known microquasar Cyg X-3 is object TeV J2032+4130. This object is still of unresolved nature and is being intensively studied in the different energy ranges. The numerous $\mathrm{X}$-ray point sources and diffuse $\mathrm{X}$-ray emission regions were found within the TeV J2032+4130 region by Chandra and Suzaku. Intensities detected in X-rays from these regions may favor a scenario with the dominantly nucleonic, not electronic origin of $\mathrm{TeV}$ emission. The results of the twenty-year observation of TeV J2032+4130 object by the SHALON experiment are presented. The collected experimental data on fluxes, spectrum shape, and morphology of TeV J2032+413 can help determine an object type and shed light on the source nature.
\end{abstract}

\section{Introduction}

Cygnus Region is one of the brightest areas of the sky in all wavelengths. It is known to contain a number of potential $\mathrm{GeV}$ and $\mathrm{TeV}$ gamma-ray sources like active star formation regions, supernova remnants (SNRs), relativistic objects. Objects connected to this region include star association Cygnus OB2, the microquasar Cygnus X-3, $\gamma$ Cygni SNR and TeV J2032+4130 object. Some of the sources listed above have been detected at high energies with Fermi LAT experiment [1-3] and earlier with EGRET telescope [4, 5]. Also, it was detected at very high energies with Milagro air shower array detector [6] and by imaging atmospheric Cherenkov telescopes Whipple [7], HEGRA [8], SHALON [9-11], VERITAS [12] and MAGIC[13]. TeV J2032+4130 was detected by the HEGRA telescopic system [8] at energies $>1 \mathrm{TeV}$ during observations made in $1999-2001$ yy. This object was the first $\mathrm{TeV}$ gamma-ray detection having no bright counterpart at any other wavelength as radio and $\mathrm{X}$-ray, and was also the first extended source to be discovered in the very high energy range.

\section{$2 \mathrm{TeV} \mathbf{J} 2032+4130$ at very high energies}

In accordance with the program on long-term studies of microquasar Cygnus X-3 [9-11] at very high energies, observations of Cygnus Region and its member objects, including TeV J2032+4130 are being carried out with the high-mountain SHALON imaging atmospheric Cherenkov telescopes since 1995.

\footnotetext{
*e-mail: sinits@sci.lebedev.ru
} 

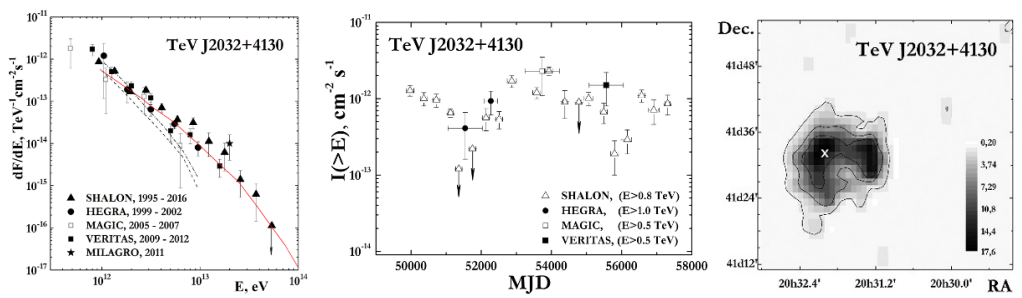

Figure 1. TeV J2032+4130 characteristics: from left to right: Differential spectrum of object by SHALON ( $\mathbf{\Lambda}$ ) in comparison with HEGRA, VERITAS, MAGIC and MILAGRO experiments;

The light curve at TeV-energies obtained in the SHALON observations $(\triangle)$ in comparison with the HEGRA, MAGIC, and VERITAS data; Emission map of TeV J2032+4130 at $>0.8 \mathrm{TeV}$ by SHALON.
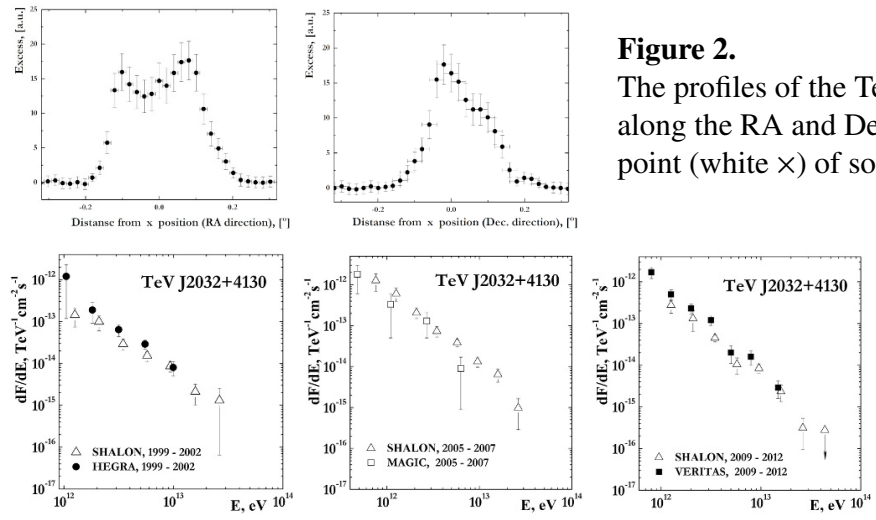

\section{Figure 2.}

The profiles of the TeV J2032+4130 emission map along the RA and Dec direction crossing through the point (white $\times$ ) of source position (Fig. 1, right).

\section{Figure 3.}

Differential spectra of $\mathrm{TeV} \mathbf{J} 2032+4130$ in different observation periods from the SHALON data compared with the HEGRA (1999 - 2002), MAGIC (2005 - 2007), and VERITAS (2009 - 2012) data.

As a result, TeV J2032+4130 was detected above $0.8 \mathrm{TeV}$ by SHALON with the average integral flux of $(0,84 \pm 0,05) \times 10^{-12} \mathrm{~cm}^{-2} \mathrm{~s}^{-1}$ at level of $31 \sigma$ determined by method [15]. The differential $\gamma$-ray energy spectrum of $\mathrm{TeV} \mathrm{J} 2032+4130$ in the energy range from 0,8 to $50 \mathrm{TeV}$ obtained by SHALON telescope is fitted well to a hard power law with an exponential cutoff: $d N / d E=(7,1 \pm 1,1) \times 10^{-13} \times\left(E_{\gamma} / 1 T e V\right)^{-1,50 \pm 0,11} \times \exp \left(-E_{\gamma} /(16 \pm 3) \mathrm{TeV}\right)$ with the $\chi^{2} / D o f=1,31$ where degree of freedom Dof $=8$. It is presented in Fig. 1 with $\Delta$ together with light-curve of this object at the period 1995 - 2015 yy. Also, the source image in TeV-energies by SHALON are shown in Figure 1. The profiles of the TeV J2032+4130 emission map along the RA and Dec direction crossing through the $\times$ point of source position are shown in Fig. 2. Analysis of the $\gamma$-shower direction showed that the emission source is an extended area of $\sim 10^{\prime}$ size, coinciding with the experimental data of HEGRA [8], VERITAS [12] and MAGIC [13].

Very high energy data on the TeV J2032+4130 energy spectrum were obtained in the different years with HEGRA [8], VERITAS [12] and MAGIC [13] ground-based telescopes and with the MILAGRO [6] detector. Figure 3 shows the comparison of differential energy spectra of TeV J2032+4130 by SHALON (1995-2016), HEGRA(1999 - 2002), MAGIC (2005 2007), VERITAS (2009 - 2012) and data of MILAGRO (2011).

The twenty-year-long SHALON observations of TeV J2032+4130 revealed flux variations at energies $>0.8 \mathrm{TeV}$. The average integral flux for TeV J2032+4130 at energies $>0.8$ TeV from the SHALON data was $(1.01 \pm 0.15) \times 10^{-12} \mathrm{~cm}^{-2} \mathrm{~s}^{-1}$ in the periods of observations 1995 - 1997, 2003 - 2006, and $2013-2015$; $(0.58 \pm 0.12) \times 10^{-12} \mathrm{~cm}^{-2} \mathrm{~s}^{-1}$ in 1998,2001 , 2002, 2010, and 2016; and a low flux, $(0.12 \pm 0.09) \times 10^{-12} \mathrm{~cm}^{-2} \mathrm{~s}^{-1}$, was recorded in 1999, 2000, 2011, and 2012. Figure 1 presents the light curve of TeV J2032+4130 obtained in the SHALON experiment over the entire period of observations from 1995 to 2015 in comparison with the HEGRA, MAGIC, and VERITAS data. The white triangles indicate the integral fluxes from the SHALON data averaged over each year of observation. 


\section{$3 \mathrm{TeV}$ J2032+413 nature}

The nature of extended object TeV J2032+413 still remains unresolved. Since the discovery of $\mathrm{TeV} \mathrm{J} 2032+4130$, several region observations have been made by X-ray telescopes Chandra, Suzaku, XMM-Newton, which operate in the energy range of $2-10 \mathrm{keV}, 0.1$ $10 \mathrm{keV}$, and $0.2-12 \mathrm{keV}$, respectively. Multiple point sources were detected in the region of TeV J2032+413 by Chandra, XMM-Newton telescopes (see Fig. 4, left), and then after the known point sources were subtracted, XMM-Newton detected an extended X-ray emission region (see $[12,19])$. Also, Suzaku detected two structures of diffuse X-ray emission in the $\mathrm{TeV} \mathrm{J} 2032+413$ region [16]. One of them coincides with the GeV old $\gamma$-ray pulsar PSR J2032+4127 detected by Fermi LAT. And overall diffuse emission of the studied region may be attributed to the IC gamma-ray origin in old PWN forming by the pulsar. Another possibility is connecting with long-period binary system forming by has mentioned gammaray pulsar PSR J2032+4127 and B0Ve star, MT91 213 having circumstellar disk of varying radius. This object lies on the edge of $\mathrm{TeV}$ emission area. In [17] spectra gamma-ray emission of the IC origin from the system are calculated, shown in the Fig. 1 with dash and dash-dot lines. In [18] studies related to the TeV J2032+413 part of Cyg OB2 massive OB association in the energy bands from radio to X-ray and simulations of the broad multifrequency demonstrated that $\mathrm{TeV}$ gamma rays are dominantly of a nucleonic, rather than an electronic, origin (see Fig. 1 with red line). On the other hand, radio observations of the region have been made using the GMRT, yielding at least three non-thermal emission sources and an extended non-thermal diffuse emission. Moreover, VLA observations revealed a shell-like structure of weak radio emission [19] consistent with the dimensions of the TeV source reported by HEGRA, VERITAS, MAGIC, and SHALON (see Fig. 4, right).
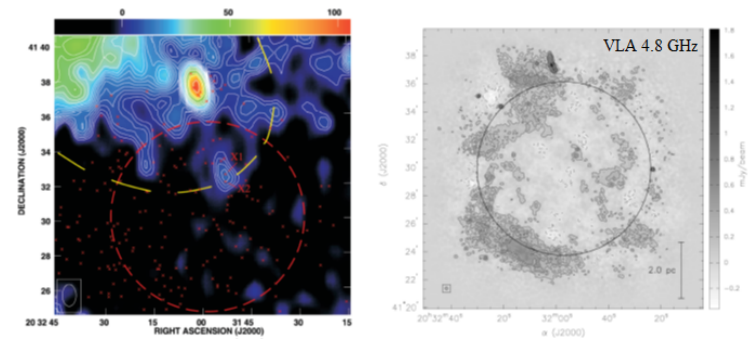

Figure 4. left: Multiple point Chandra X-ray sources (red crosses) were detected in the region of $\mathrm{TeV}$ J2032+413 marked with red dashed circle line;

right: Shell-like structure of weak radio emission observed by VLA.(pictures adopted from [19]) $\mathrm{TeV} \mathrm{J} 2032+413$ source is indicated by the overlaid black line circle)

\section{Conclusions}

The results of long-term observations of TeV J2032+4130 object by imaging atmospheric Cherenkov telescope SHALON are presented. Characteristics of TeV J2032+4130 in the energy range from 1 up to $50 \mathrm{TeV}$ were obtained with SHALON telescope for the first time. The collected experimental data and refining of fluxes, spectrum shape, and morphology of TeV J2032+413 favor the nucleonic origin of the observed emission and can help in the future determine an object type on the source and shed light on its nature.

\section{References}

[1] A.A. Abdo, B. Allen, D. Berley et al., Astrophys. J. 658, L33 (2007)

[2] A.A. Abdo, M. Ackermann, M. Ajello et al., Science 326, 1512 (2009)

[3] A.A. Abdo, M. Ackermann, M. Ajell et al., Astrophys. J. 187, 460 (2010)

[4] D.J. Thompson et al., Astrophys. J. Suppl. Ser. 101, 259 (1995)

[5] J.A. Esposito, S.D. Hunter, G. Kanbach, P. Sreekumar, Astrophys. J. 461, 820 (1996) 
[6] A.A. Abdo, B. Allen, T. Aune et al., Astrophys. J. 734, 28 (2011)

[7] J.H. Buckley et al., Astron.\& Astrophys. 329, 639 (1998)

[8] F. Aharonian, A. Akhperjanian, M. Beilicke et al., Astron.\& Astrophys. 431, 197 (2005)

[9] V.G. Sinitsyna, in Proc. 16th European Cosmic Ray Symposium, ed. J. Medina, (Alcala de Henares: Universidad de Alcala, Spain, 1998), 383

[10] V.G. Sinitsyna \& V.Y. Sinitsyna, Bulletin of the Lebedev Physics Institute, (New York: Allerton Press, Inc.), 40(5),113 (2013)

[11] V.G. Sinitsyna \& V.Y. Sinitsyna, EPJ Web of Conferences 145 04002, (2017)

[12] E. Aliu, T. Aune, B. Behera et al., Astrophys. J. 783, 16 (2014)

[13] J. Albert, E. Aliu, H. Anderhub et al., Astrophys. J. 675, L25 (2008)

[14] V.G. Sinitsyna, Nuovo Cimento 19C(6), 965 (1996)

[15] T.-P. Li \& Y.-Q. Ma, Astrophys. J. 272, 317 (1983)

[16] H. Murakami \& S. Kitamoto, Publ. Astron. Soc. Japan 63, S873 (2011)

[17] W. Bednarek, P. Banasiński, J. Sitarek, JPhG 45, 015201 (2018)

[18] Y.M. Butt, P. Benaglia, J.A. Combi et al.,Astrophys. J. 597, 494 (2003)

[19] Y.M. Butt, J.A. Combi, J. Drake et al., Mon. Not. Roy. Astron. Soc. 385, 1764 (2008) 